\title{
Kinematics Research of Rotating-Sleeve Distributing-Flow System for Reciprocating Plunger Pump
}

\author{
Xu Wei ${ }^{1}, a^{*}$, Zhang Hongxin ${ }^{1, b}$, Shu Pei ${ }^{1, c}$, Gao Lei ${ }^{1, d}$ \\ ${ }^{1} 308$ Ningxia Road, Qingdao, Shandong, P.R.C, 266071 \\ aqduxuwei@foxmail.com, bqduzhx@126.com, qdushupei1988@126.com, 'dgaoleiqdu@163.com
}

\begin{abstract}
Key words: distributing-flow system; reciprocating plunger pump; cylindrical cam; kinematics Abstract. The traditional valve orifice system had some shortages like: loose structure, more throttling loss, low efficiency and affected highly by operating frequency, then proposed and adopted a new type of rotating-sleeve distributing-flow system for reciprocating plunger pump. Established the kinematic characteristic of reciprocating plunger pump, researched the motion characteristics of reciprocating plunger pump. It provides the theoretical foundation for the following research.
\end{abstract}

\section{Introduction}

Reciprocating plunger pump widely used in oil transmission, pump station, hydraulic engine and large machinery, the flow allocation of reciprocating plunger pump mainly adopts valve type. Compared with the end surface of flow allocation, the advantage of valve type is to reduce the friction of the cylinder and valve plate, thereby reducing wear and leakage. But the reciprocating plunger pump also has some shortcomings: First, the structure is bulky and loose, it is difficulty to mounting, especially in pipe joints, and it is easy to leaks; Second, the local throttling losses of one-way valve and switching valve is large; Third, the volumetric efficiency of the pump influenced by the work frequency obviously, once deviated its calibration conditions, the volumetric efficiency will reduce significantly. Thus, the rotating-sleeve distributing-flow system for reciprocating plunger pump that used in the Gear-linked Cylinders Hydraulic engine is proposed.

\section{Structure and working principle}

As the hydraulic chamber of GCHE, the structural principle of distributing-flow system is shown in Fig.1. It guides reciprocating linear motion into continuous unidirectional rotary motion of rotating-sleeve by a direct-acting cylinder cam mechanism, and uses the unidirectional rotary motion of the sleeve to achieve flow allocation.

The depth of CAM slot in inner wall of rotating-sleeve is metabolic, the rotating-sleeve and plunger connects by drive pin, drive pin always compressing the rotating-sleeve under the pressure of spring. When the plunger moves from BDC to TDC, the rotating-sleeve rotates 180 degree and the depth of CAM slot become shallow. When the plunger moves from TDC to BDC, the rotating-sleeve also rotates 180 degree and the depth of CAM slot become shallow. The rotating-sleeve always rotates unidirectional because of the step change of the depth of CAM slot at $\mathrm{BDC}$ and TDC. 


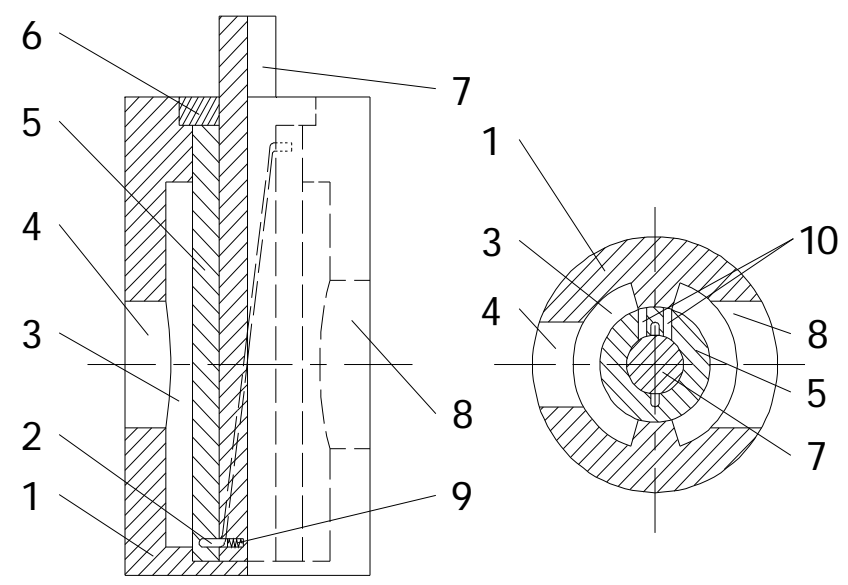

1. Pump shell; 2. Drive pin; 3 Pump chambers; 4. Liquid inlet; 5. Rotating-sleeve; 6. Sleeve; 7. Plunger; 8. Liquid outlet; 9. Spring; 10. Flow Allocation mouth

Fig.1. Cutaway view of the rotating-sleeve reciprocating plunger pump

\section{Kinematics analysis of the rotating-sleeve reciprocating plunger pump}

The cam slot line equation of the rotating-sleeve can be expressed as $r=r(\theta), z=z(\theta)$ by column coordinate and the equation should satisfy the following conditions: The radial velocity of drive pin is zero, when the plunger at BDC and TDC, and the first derivative of $r(\theta)$; For $z(\theta)$, when $\theta=\pi, z=S$; when $\theta=2 \pi, \mathrm{z}=0$. The radial and axial equations of the slot line are:

$$
\begin{aligned}
& r(\theta)=\left\{\begin{array}{c}
d / 2+h_{\text {max }}-3 h(\theta / \pi)^{2}+2 h(\theta / \pi)^{3}(0 \leq \theta<\pi) \\
d / 2+h_{\max }-3 h[(\theta-\pi) / \pi]^{2}+2 h[(\theta-\pi) / \pi]^{3}(\pi \leq \theta \leq 2 \pi)
\end{array}\right. \\
& z(\theta)=\left\{\begin{array}{cl}
\theta \cdot S / \pi & (0 \leq \theta<\pi) \\
2 S-\theta \cdot S / \pi & (\pi \leq \theta \leq 2 \pi)
\end{array}\right.
\end{aligned}
$$

The rotating-sleeve is an important flow part of the plunger pump, the rotation rule is affected by the crank angle of GCHE, and the rotation equation is:

$$
\theta(\varphi)= \begin{cases}\frac{\pi}{2 \lambda}\left(1+\lambda-\lambda \cos \varphi-\sqrt{1-\lambda^{2} \sin ^{2} \varphi}\right) & (0 \leq \varphi \leq \pi) \\ 2 \pi-\frac{\pi}{2 \lambda}\left(1+\lambda-\lambda \cos \varphi-\sqrt{1-\lambda^{2} \sin ^{2} \varphi}\right) & (\pi \leq \varphi \leq 2 \pi)\end{cases}
$$

Find the first differential and second differential of eq. (3). The equations of angular velocity and angular acceleration are:

$$
\begin{aligned}
& \omega_{r}(\varphi)= \pm \frac{\pi \omega}{2}\left(\sin \varphi+\frac{1}{2} \lambda \sin 2 \varphi / \sqrt{1-\lambda^{2} \sin ^{2} \varphi}\right) \\
& \alpha_{r}(\varphi)= \pm \frac{\pi \omega^{2}}{2}\left\{\cos \varphi+\left[4 \lambda \cos 2 \varphi\left(1-\lambda^{2} \sin ^{2} \varphi\right)+\lambda^{3} \sin ^{2} 2 \varphi\right] /\left(1-\lambda^{2} \sin ^{2} \varphi\right)^{\frac{3}{2}}\right\}
\end{aligned}
$$

The sign of eq. (4) and eq. (5) is plus, when $0 \leq \varphi<\pi$, and the sign is subtraction, when $\pi \leq \varphi \leq 2 \pi$.

Drive pin is the main force transmission part of plunger pump. Substituting eq. (3) into eq. (1), the radial equation of the slot that changes with the crank angle can be described as:

$$
r(\varphi)=\left\{\begin{array}{l}
\frac{d}{2}+h_{\max }+\frac{h}{4 \lambda^{2}}\left(1+\lambda-\lambda \cos \varphi-\sqrt{1-\lambda^{2} \sin ^{2} \varphi}\right)^{2}\left(1-2 \lambda-\lambda \cos \varphi-\sqrt{1-\lambda^{2} \sin ^{2} \varphi}\right)(0 \leq \varphi<\pi) \\
\frac{d}{2}+h_{\max }-\frac{h}{4 \lambda^{2}}\left(1-\lambda-\lambda \cos \varphi-\sqrt{1-\lambda^{2} \sin ^{2} \varphi}\right)^{2}\left(1+2 \lambda-\lambda \cos \varphi-\sqrt{1-\lambda^{2} \sin ^{2} \varphi}\right)(\pi \leq \varphi \leq 2 \pi)
\end{array}\right.
$$


Find the first differential and second differential of eq. (6). The velocity equation and acceleration equation of drive pin are:

$$
\begin{aligned}
& v_{r}(\varphi)= \pm \frac{3 h \omega}{4 \lambda^{2}}\left(\sin \varphi+\frac{1}{2} \lambda \sin 2 \varphi / \sqrt{1-\lambda^{2} \sin ^{2} \varphi}\right)\left[\left(1-\lambda \cos \varphi-\sqrt{1-\lambda^{2} \sin ^{2} \varphi}\right)^{2}-\lambda^{2}\right] \\
& a_{r}(\varphi)= \pm \frac{3 h \omega^{2}}{2 \lambda^{2}}\left(\sin \varphi+\frac{1}{2} \lambda \sin 2 \varphi / \sqrt{1-\lambda^{2} \sin ^{2} \varphi}\right)^{2}\left(1-\lambda \cos \varphi-\sqrt{1-\lambda^{2} \sin ^{2} \varphi}\right)
\end{aligned}
$$

The sign of eq. (4) and eq. (5) is plus, when $0 \leq \varphi<\pi$, and the sign is subtraction, when $\pi \leq \varphi \leq 2 \pi$. Table 1 presents the definition and value of symbols used in the simulation.

\begin{tabular}{l|l|l}
\hline Symbol & Definition & Value \\
\hline$d$ & plunger diameter(m) & $2.1 \times 10^{-2}$ \\
\hline$h$ & depth changes of the cam groove $(\mathrm{m})$ & $5 \times 10^{-3}$ \\
\hline$h_{\max }$ & maximum depth of the cam groove $(\mathrm{m})$ & $8 \times 10^{-3}$ \\
\hline$S$ & plunger stroke(m) & $1.35 \times 10^{-1}$ \\
\hline$\varphi$ & crank angular position(rad) & - \\
\hline$\omega$ & crank turning speed(rad/s) & $1.99 \times 10^{2}$ \\
\hline$\lambda$ & crankshaft ratio & 0.3125 \\
\hline
\end{tabular}

\section{Results}
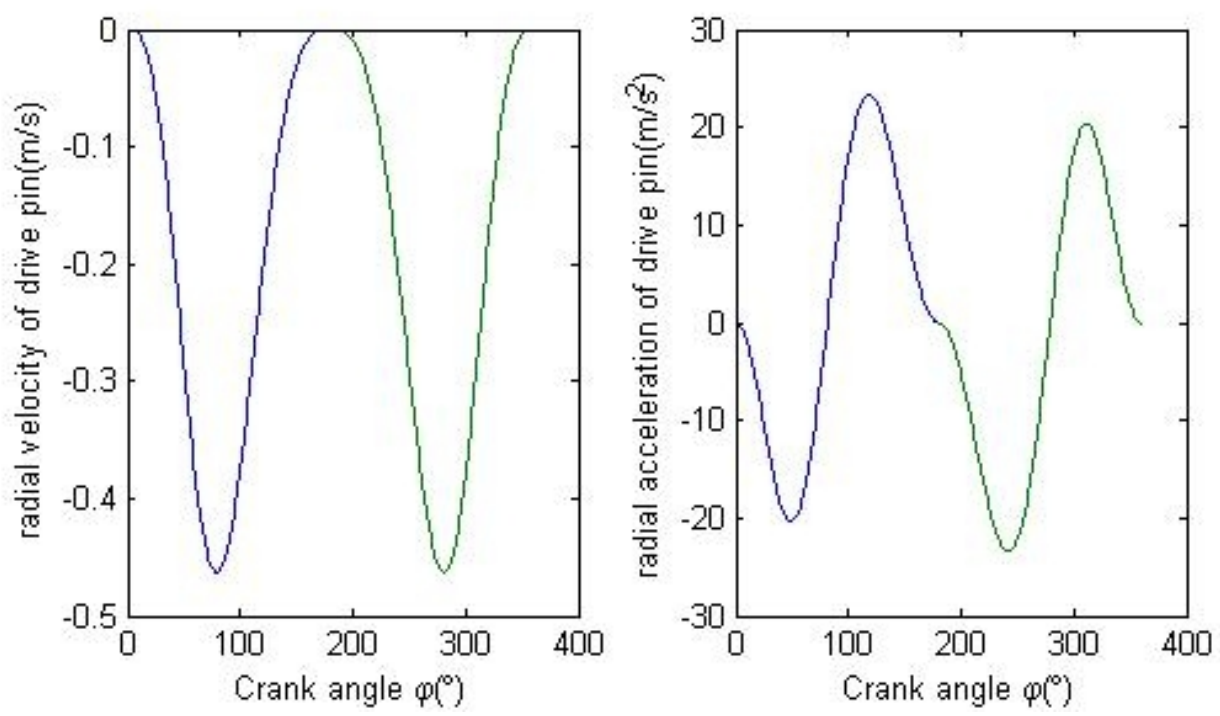

Fig. 2 The radial velocity and acceleration of drive pin

Fig. 2 shows the radial velocity and radial acceleration of drive pin, defining away from radial as a plus sign. It is observed that the maximum velocity is $0.4626 \mathrm{~m} / \mathrm{s}\left(\varphi=79^{\circ}\right)$, the maximum acceleration is $23.37 \mathrm{~m} / \mathrm{s}^{2}\left(\varphi=118^{\circ}\right)$, the period of velocity and acceleration is $\pi$.

Fig. 3 shows the angular velocity and angular acceleration of rotating-sleeve, defining Clockwise rotation as a plus sign. Fig. 3 shows the maximum angular velocity is $352.2 \mathrm{rad} / \mathrm{s}\left(\varphi=66^{\circ}\right.$ and $\left.294^{\circ}\right)$, the maximum angular acceleration is $1.4 \times 10^{5} \mathrm{rad} / \mathrm{s}^{2}\left(\varphi=0^{\circ}\right)$. 

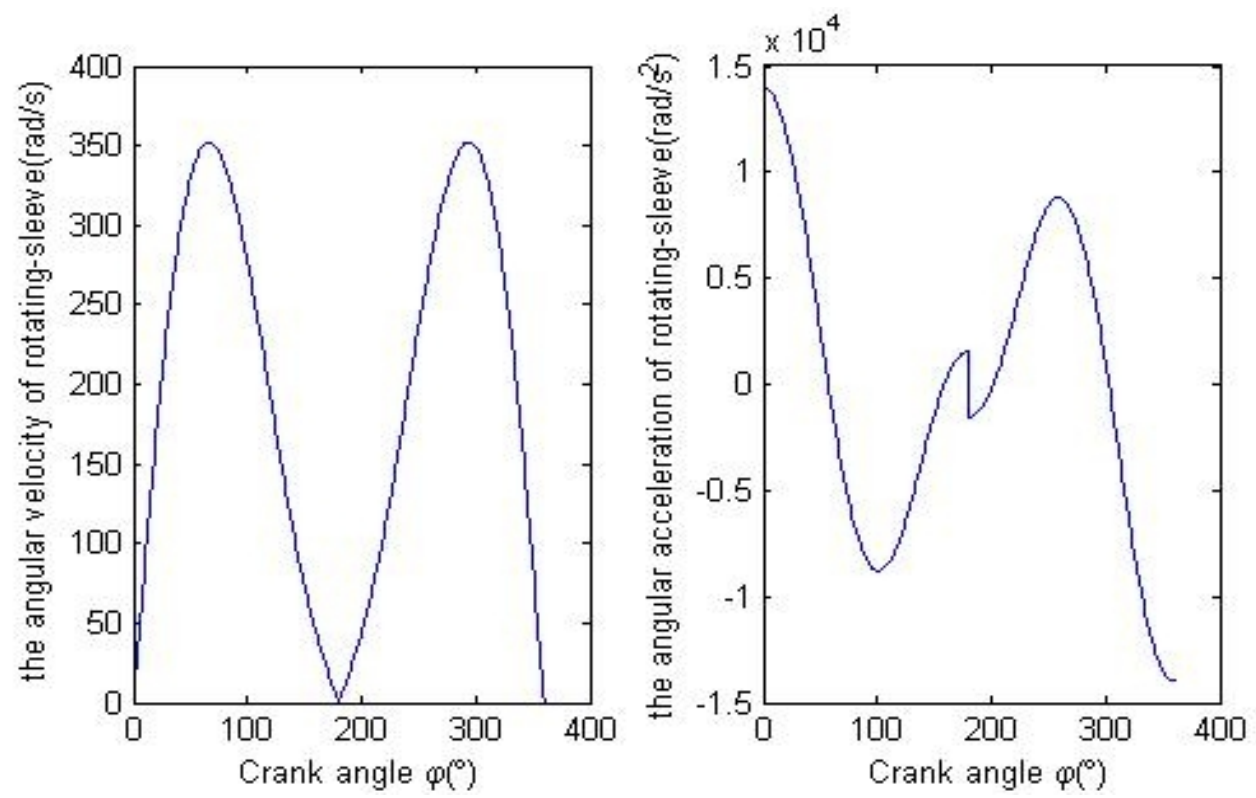

Fig. 3 The angular velocity and angular acceleration of rotating-sleeve

\section{Conclusions}

Established the kinematics model of the rotating-sleeve reciprocating plunger pump, obtained the motion parameters of each moving parts, and provided a theoretical basis for follow-up design and optimization design.

\section{Acknowledgements}

The authors gratefully acknowledge the financial support from the National Natural Science Foundation (No.51575286) and Shandong Provincial Science Foundation of the P. R. of China (No. 2014ZRB01503)

\section{References}

[1] Lu Yongxiang. Hydraulic and pneumatic Technical Manual [M]. Beijing: Mechanical Industry Press, 2007

[2] Zhang Hongxin, Zhang Tiezhu. A kind of Gear-linked Cylinders Hydraulic engine [P]. China, CN201310015184.4, 2013-04-03 .

[3] Zhang Hongxin, Shu pei. A kind of Rotating-Sleeve Distributing-Flow System for Reciprocating Plunger Pump [P]. China, CN201310706745.5 , 2014-03-12

[4] Zhang Hongxin, Zhang Tiezhu, Zhao hong. Simulation Study on Working Process of Single-cylinder Axial Internal Combustion Engine [J] Fluid Machinery, 2007, 35(3):1-5

[5] Shu pei, Zhang Hongxin, Xiao Ruqin. Structure Design and Research of the Gear-linked Cylinders Hydraulic Engine [J] Modern Manufacturing Engineering.2015, 5:53-57 